\title{
Association of HLA-DRB1 and DQB1 alleles with red blood cell alloimmunization in Chinese
}

\author{
Yu-Shiang $\operatorname{Lin}^{1,2 \Delta}$, Zhiyuan $\mathrm{Xu}^{3 \Delta}$, Haochun Chang ${ }^{1,2}$, Xiaofei $\mathrm{Li}^{3}$, Daowong $\mathrm{Fan}^{3}$, Yan Qiu ${ }^{3}$, Tianhong Miao ${ }^{3 *}$ \\ ${ }^{1}$ Department of Clinical Medicine, School of Basic Medical Sciences, Peking University Health Science Center, Beijing, 100191, China; \\ ${ }^{2}$ College of Medicine, Aerospace Center Hospital, Beijing, 100076, China; \\ ${ }^{3}$ Blood Group Lab, Beijing Red Cross Blood Center, Beijing, 100088, China
}

\begin{abstract}
Few systematic investigations have assessed the correlations between red blood cell (RBC) antibodies and human leukocyte antigen (HLA)-DRB1 alleles in the Chinese population. In this case-control study, we investigated whether specific HLA-DRB1 alleles were associated with RBC alloimmunization by calculating the odds ratios for the frequencies of HLA alleles associated with alloimmunization to different RBC antigens. Three hundred and eight patients harboring RBC alloantibodies were analyzed as the case group, and the frequencies of the HLA-DRB1 and HLA-DQB1 alleles in control individuals were analyzed by collecting data from the China Marrow Donor Program (including more than 1.6 million healthy people). HLA alleles were genotyped by single specific primer-polymerase chain reaction. The development of anti-C was associated with $\mathrm{DRB1} * 07, \mathrm{DQB} 1 * 06$, and DQB1*08; anti-C, e was associated with $\mathrm{DRB1} * 07$ and $\mathrm{DQB1} * 06$; anti-E and anti-M were associated with DQB1. Other associations were identified between anti-E and DRB1*09 and between anti-Le ${ }^{\mathrm{a}}$ and DRBI*01. Thus, our findings confirmed that HLA-DRBI and DQBl restriction played an important role in the generation of RBC alloantibodies in Chinese individuals.
\end{abstract}

Keywords: human leukocyte antigen-DRB1, human leukocyte antigen-DQB1, red blood cell alloantibody

\section{INTRODUCTION}

The development of red blood cell (RBC) alloantibodies (RAAbs) may be stimulated by transfusion, pregnancy, and transplantation ${ }^{[1-3]}$. The incidence of RAAbs has been reported to range from $1 \%$ to $3 \%$, and patients who receive multiple transfusions have been shown to have a prevalence as high as $1 \%-6 \%{ }^{[4-6]}$. The variations in the rates of RAAbs among patients may be explained by differences in regular follow-up testing after transfusion. However, the cause of RAAbs in some patients who have received transfusions is still unclear, and few studies have examined differences in
RAAb rates.

The risk factors for RAAbs include older age, female sex, blood transfusion, and human leukocyte antigen (HLA) class II expression. Schonewille et al. confirmed that an association with DRB1*15 was present in almost $40 \%$ of cases, compared with approximately $25 \%$ in single-antibody responders, and identified the relationships between anti-E and DRB1*09 and between anti-S and DRBI*07 in European populations ${ }^{[7]}$. Maluskova et al. observed an association between HLA-DRB1*15 and RBC antibody multi-responder status and found HLA-class II associations for three frequent RBC antibody combinations in the Czech population ${ }^{[8]}$. Additionally,

*Correspondence to: Tianhong Miao, Blood Group Lab, Beijing Red Cross Blood Center, Beijing, 100088, China. TEL: +86-10-82807272; E-mail: tianhongm@163.com.

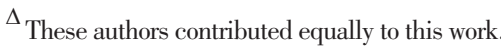

The authors declared no conflict of interests. 
we reported HLA-DRBI*13 as a protective factor in RBC autoimmunization ${ }^{[9]}$. However, few studies have observed associations between HLA class II alleles and RAAbs in the Chinese population ${ }^{[10]}$. Notably, in contrast to European, American, and African populations, anti-E was the most prevalent form, followed by anti-M, anti-C, e, and anti-Le ${ }^{\mathrm{a}}$, in the Chinese population. The HLA frequencies also varied among ethnic groups.

In clinical transfusion practice, several studies have demonstrated associations between HLA-DRBI and RBC alloantibodies and confirmed the effects of different HLA-DRBI alleles on multi-responders, specific alloantibodies, and immunization following a single transfusion ${ }^{[1,11]}$. Only one study examined the relationships between HLA-DRB1/DQB1 and RBC autoimmunization, and no reports have described the HLA class II status in RBC alloantibodies. Therefore, in this study, we aimed to investigate the associations between HLA-DRB1/HLA-DQB1 and RAAbs in Chinese individuals in order to determine the HLA status of RBC immunization, and identify biomarkers of safe transfusions.

\section{MATERIALS AND METHODS}

\section{Study population}

The patients who needed RBC transfusion with antibody screen test positive were taken from North Blood Grouping Reference Lab(BGRL) of China, Beijing Red Cross Blood Center (BRCBC) from Dec. 2015 to Dec. 2016. All 308 patients were identified as RAAbs via plasma clump with all screen cells and panel cells, and HLA-DRB1 and DQB1 genotyping were analyzed for all subjects.

The 308 patients with RAAbs were grouped as anti-M, anti-Le ${ }^{\mathrm{a}}$, anti-C, anti-C, e, anti-D, anti-E and anti-c, E base. To observe an association between HLA alleles with more than 2 RBC antibodies, comparisons were done on two-antibody combinations per time. For example, for a patient with anti$\mathrm{C}$, e, the frequencies of combined associated HLA alleles for anti-C, anti-e, and anti-C, e were compared to the controls. For all subgroups, frequencies of HLA-DRB1 and HLA-DQB1 were calculated and compared to alleles frequency from the China Bone Marrow Donor Registry Program (CBMDRP), which is able to draw data from over 1.6 million Chinese participants ${ }^{[7]}$. We set the case control study to count odds ratio (OR) with $95 \%$ confidence interval (CI) for quantization of risks or protection and the case control ratio was 1:4. Our statistical procedures are similar to our previous study ${ }^{[7]}$.

\section{Antibody screen test, direct antiglobulin test and antibody identification}

Screen and identification cells used at the BGRL of the BRCBC were designed to cover antigen systems, including Rh, MNS, Duffy, Kidd, Kell, Lewis, P1, $\mathrm{Xg}$, and Lutheran, as well as low-frequency antigens such as Dia and Mur (Bio-RAD GmbH, Germany and Sanquin GmbH, Switzerland), which have a relatively high prevalence in Asia. Detection methods include conventional tube technique in room temperature and gel column tests (Bio-Rad GmbH, Germany). Only a few individual samples that showed negative results in the gel column test were identified positive in the room temperature tube test for antibody identification. The other procedures such as phenotyping, sample reception, report documentation, and report writing were all based on regulations and the standard procedures specified by the BGRL of the BRCBC.

\section{DNA extraction and genotyping of HLA-DRB1 and HLA-DQB1}

DNA was isolated from EDTA-anticoagulated blood using a commercial kits (Prepito DNA Blood 250 Kit, Chemagen, PerkinElmer, Germany) based on chemagic magnetic separation in automatic equipment (Chemagic Prepito, Chemagen, PerkinElmer, Germany). All DNA samples were stored at $-80^{\circ} \mathrm{C}$ until molecular analysis. High-resolution DRB1-SSP, DQB1-SSP commercial kits (LABType SSO, One Lambda, Canoga Park, CA) are used for HLA-DRB1 and HLA-DQB1 genotyping.

\section{Statistical analysis}

Chi-square and Fisher exact tests were used to evaluate comparisons between the study groups. Bonferroni formula [adjusted $P$ value $\left.=1-(1-\text { crude } P)^{\mathrm{n}}\right]$ was corrected for multiple testing, and adjusted $P\left(P^{\mathrm{a}}\right)$ with a 2 -sided $P$ value $<0.05$ were considered statistically significant. All statistical analyses were conducted by using SPSS version 19.0 (SPSS Inc., Chicago, IL, USA).

\section{RESULTS}

To identify the associations of specific unexpected RBC antibodies with HLA-class II variants, 308 patients (101 men and 207 women) of Chinese descent were enrolled in this study. Of these patients, 73 formed anti-E, 50 formed anti-M, 50 formed anti-Le ${ }^{\mathrm{a}}, 33$ formed anti-C, e, 30 formed anti-c, E, 28 formed antiC, D, 16 formed anti-D, 13 formed anti-C, 6 formed anti-D, E, 4 formed anti-c, E, autoantibodies, 4 formed anti-c, E, Jk ${ }^{\mathrm{b}}$, and 1 formed anti-C, S. As shown in 
Table 1, 117 samples were classified as anti-E group; 75 samples were classified as anti-C group; 50 samples were classified in each of the anti-Le ${ }^{\mathrm{a}}$, anti-M, and
anti-D groups; 38 samples were classified as anti-c, E; 33 samples were classified as anti-C, e; and 28 samples were classified as anti-C, D.

Table 1 Baseline of patients with RAAbs

\begin{tabular}{|c|c|c|c|c|c|c|c|c|c|c|}
\hline RAAbs & Age/year,mean \pm SD & Sex/male, $n(\%)$ & Anti-M & Anti-Le ${ }^{\mathrm{a}}$ & Anti-E & Anti-D & Anti-C & Anti-C, D & Anti-c, E & Anti-C, e \\
\hline Anti-M $(n=50)$ & $49.4 \pm 21.4$ & $16(32.0)$ & 50 & & & & & & & \\
\hline $\operatorname{Anti}-\operatorname{Le}^{\mathrm{a}}(n=50)$ & $43.4 \pm 18.1$ & $10(20.0)$ & & 50 & & & & & & \\
\hline Anti-E( $(n=73)$ & $53.5 \pm 17.9$ & $23(31.5)$ & & & 73 & & & & & \\
\hline Anti-D $(n=16)$ & $47.3 \pm 27.6$ & $6(37.5)$ & & & & 16 & & & & \\
\hline Anti-C $(n=13)$ & $60.8 \pm 24.5$ & $8(61.5)$ & & & & & 13 & & & \\
\hline Anti-C, D(n=28) & $47.0 \pm 39.6$ & $11(39.3)$ & & & & 28 & 28 & 28 & & \\
\hline Anti-c, $\mathrm{E}(n=30)$ & $56.1 \pm 13.6$ & $12(40.0)$ & & & 30 & & & & 30 & \\
\hline Anti-C, e $(n=33)$ & $48.0 \pm 23.8$ & $33(39.4)$ & & & & & 33 & & & 33 \\
\hline Anti-C, $\mathrm{S}(n=1)$ & 70.1 & $0(0.0)$ & & & & & 1 & & & \\
\hline Anti-D, E $(n=6)$ & $39.3 \pm 9.2$ & $2(33.3)$ & & & 6 & 6 & & & & \\
\hline Anti-c, E, Jk ${ }^{\mathrm{b}}(n=4)$ & $59.0 \pm 5.8$ & $0(0.0)$ & & & 4 & & & & 4 & \\
\hline $\begin{array}{l}\text { Anti-c, E, } \\
\text { autoantibodies }(n=4)\end{array}$ & $50.0 \pm 8.1$ & $0(0.0)$ & & & 4 & & & & 4 & \\
\hline $\operatorname{Total}(n=308)$ & $20.5 \pm 22.3$ & $101(32.8)$ & 50 & 50 & 117 & 50 & 75 & 28 & 38 & 33 \\
\hline
\end{tabular}

\section{HLA-DRB1 frequencies in patients with spe- cific $\mathbf{R B C}$ alloantibodies}

The frequencies of HLA-DRB $* 01$, HLADRB $1 * 04$, HLA-DRB $1 * 07$, HLA-DRB $1 * 09$, and HLA-DRB ${ }^{*} 13$ were higher among subjects with various types of RBC antibody specificities than in the CBMDRP. After adjusting the $P$ values using
Bonferroni analysis, patients with anti-C or anti-C, e showed a higher frequency of HLA-DRB1 $* 07$ (OR: 2.11, 95\%CI: 1.39-3.22 for anti-C; OR: 3.00, 95\%CI: 1.73-5.19 for anti-C, e, Table 2). Additionally, those with anti-Le ${ }^{\mathrm{a}}$ showed a higher frequency of HLADRBI*01 (OR: 4.44, 95\%CI: 1.86-10.65), and those with anti-E showed a higher frequency of HLADRB1*09 (OR: 2.00, 95\%CI: 1.09-3.66).

Table 2 HLA-DRB1 associations for patients with RAAbs

\begin{tabular}{lcccccccc}
\hline RAAbs & Alleles & Case $/ n$ & Case/\% & Control/\% & ORs & 95\%CI & Crude $P$ & Adjusted $P$ \\
\hline Anti-C $(n=150)$ & DRB1*07 & 28 & 18.7 & 8.9 & 2.11 & $1.39-3.22$ & $<0.001$ & $<0.001$ \\
Anti-C, e $(n=66)$ & DRB1*07 & 18 & 27.3 & 8.9 & 3.00 & $1.73-5.19$ & $<0.001$ & $<0.001$ \\
Anti-c,E $(n=76)$ & DRB1*07 & 16 & 18.6 & 8.9 & 2.10 & $1.18-3.71$ & 0.018 & 0.196 \\
Anti-C, D $(n=56)$ & DRB1*14 & 11 & 19.6 & 7.0 & 2.75 & $1.35-5.59$ & 0.009 & 0.103 \\
Anti-D $(n=100)$ & DRB1*04 & 22 & 22.0 & 11.3 & 1.96 & $1.23-3.10$ & 0.008 & 0.092 \\
Anti-E $(n=234)$ & DRB1*04 & 38 & 16.2 & 11.3 & 1.43 & $1.02-2.02$ & 0.008 & 0.092 \\
& DRB1*07 & 35 & 15.0 & 8.9 & 1.69 & $1.17-2.44$ & 0.008 & 0.092 \\
& DRB1*09 & 63 & 26.9 & 14.3 & 1.88 & $1.45-2.45$ & $<0.001$ & $<0.001$ \\
Anti-Le $(n=100)$ & DRB1*01 & 10 & 10.0 & 2.3 & 4.44 & $1.86-10.65$ & 0.001 & 0.012 \\
Anti-M $(n=100)$ & DRB1*14 & 14 & 14.0 & 7.0 & 2.00 & $1.09-3.66$ & 0.041 & 0.395 \\
\hline
\end{tabular}

\section{HLA-DQB1 frequencies in patients with spe- cific RBC alloantibodies}

Anti-C and anti-C, e groups showed increased frequencies of HLA-DQB1*06 (OR: 1.55, 95\%CI: 1.192.01 for anti-C; OR: 1.93, 95\%CI: 1.36-2.75 for anti$\mathrm{C}$, e, Table 3). Additionally, the anti-C group showed an increased frequency of HLA-DQB1*08(OR: 2.47, 95\% CI: 1.48-4.13). Interestingly, the anti-E group showed a high frequency of HLA-DQB $1 * 08$ (OR: $3.11,95 \% \mathrm{CI}: 2.13-4.54)$, as did the anti-M group (OR: 2.61, 95\%CI: 1.41-4.81).

\section{DISCUSSION}

In the present study, we observed the possible associations between HLA-DRB1*13 as protective factor and RBC autoantibodies ${ }^{[9]}$. This study is the first to demonstrate the presence of RBC-specific alloantibodies with HLA class II in Chinese patients. Prior to this, few studies have systematically observed the associations between specific alloantibodies and HLA class II typing, particularly HLA-DQB1 typing in Chinese individuals. In a Chinese study, Wu et al. reported that HLA-DRB1*07:01 was associated with 
Table 3 HLA-DQB1 associations for patients with RAAbs

\begin{tabular}{|c|c|c|c|c|c|c|c|c|}
\hline RAAbs & Alleles & Case $/ n$ & Case $/ \%$ & Control/\% & OR & $95 \% \mathrm{CI}$ & Crude $P$ & Adjusted $P$ \\
\hline \multirow[t]{2}{*}{ Anti-C $(n=150)$} & $\mathrm{DQB} 1 * 06$ & 53 & 35.3 & 22.8 & 1.55 & $1.19-2.01$ & 0.002 & 0.016 \\
\hline & $\mathrm{DQB} 1 * 08$ & 21 & 14.0 & 5.7 & 2.47 & $1.48-4.13$ & 0.001 & 0.008 \\
\hline Anti-C, e $(n=66)$ & DQB $1 * 06$ & 29 & 43.9 & 22.8 & 1.93 & $1.36-2.75$ & $<0.001$ & $<0.001$ \\
\hline Anti-c, $\mathrm{E}(n=76)$ & DQB1*08 & 11 & 14.5 & 5.7 & 2.59 & $1.27-5.29$ & 0.013 & 0.099 \\
\hline Anti-D $(n=100)$ & DQB1*08 & 21 & 21.0 & 5.7 & 3.65 & $2.11-6.33$ & $<0.001$ & \\
\hline \multirow[t]{2}{*}{ Anti-E $(n=234)$} & $\mathrm{DQB} 1 * 02$ & 44 & 18.8 & 12.5 & 1.50 & $1.10-2.06$ & 0.015 & 0.114 \\
\hline & $\mathrm{DQB} 1 * 08$ & 42 & 17.9 & 5.7 & 3.11 & $2.13-4.54$ & $<0.001$ & $<0.001$ \\
\hline $\operatorname{Anti}^{-L^{a}}(n=100)$ & $\mathrm{DQB} 1 * 08$ & 14 & 14.0 & 5.7 & 2.44 & $1.30-4.56$ & 0.009 & 0.070 \\
\hline \multirow[t]{2}{*}{ Anti-M(n=100) } & $\mathrm{DQB} 1 * 04$ & 12 & 12.0 & 5.7 & 2.09 & $1.08-4.05$ & 0.045 & 0.308 \\
\hline & DQB1*08 & 15 & 15.0 & 5.7 & 2.61 & $1.141-4.81$ & 0.005 & 0.039 \\
\hline
\end{tabular}

the production of alloantibodies against $\mathrm{RhD}^{[12]}$. In a previous study in the Netherlands, HLA-DRB1* 15 was found to enhance the formation of multiple RBC antibody specificities ${ }^{[7]}$, and Maluskova et al. confirmed the association of HLA-DRB1*15 with RBC antibody multi-responder status in the Czech population $^{[8]}$.

The significant association between HLADRB1*09 and anti-E in our study was inconsistent with a European study in which Schonewille et al. reported that anti-Fy ${ }^{\mathrm{a}}$, anti-D, anti-C, and anti-K were associated with HLA-DRB1*1 $15^{[9,13]}$. Notably, in the Chinese population with anti-E, the frequencies of $\mathrm{Fy}^{\mathrm{a}}$ and D antigens were higher than $99 \%$, and that of $\mathrm{K}$ antigen was much lower (less than $1 \%$ ); this antigen frequency led to the reduced presence of alloantibodies against $\mathrm{K}, \mathrm{Fy}^{\mathrm{a}}$, and D antigens. However, in our study, anti-C was present at a higher frequency in patients with $\mathrm{HLA}-\mathrm{DRB} 1 * 07$, and HLA-DQB1*06 also was a risk factor for immunity to anti-C and anti-C, e. Our study was also the first to show that anti- $\mathrm{Le}_{\mathrm{a}}$ was associated with HLA-DRB $1 * 01$.

Only one study showed that HLA-DQBI is related to RBC alloantibodies. Maluskova et al. demonstrated that $\mathrm{DQB1} 1 * 06$ was more frequent in multi-responders with anti-E+c and that $\mathrm{DQB} 1 * 02$ was more frequent in those with anti-E+C $C^{[8]}$. Our findings showed that HLA-DQB1 $* 06$ was associated with anti-C and anti$\mathrm{C}$, e and that HLA-DQB1*08 was related to anti-E and anti-M. In general, HLA class II plays an important role in the immune response, and many studies have confirmed that HLA class II molecules are related to transplantation integrity, vaccine response, and viral infection. A meta-analysis demonstrated that $\mathrm{DQB1}{ }^{*} 02: 01$ and $\mathrm{DQB} 1 * 03: 03$ were risk factors for decreased titers for the Mumps-Measles-Rubella and hepatitis B virus vaccines ${ }^{[14-17]}$. Our study not only confirmed that HLA-DRB1 was associated with RBC alloantibodies but also revealed that HLA-DQB1 was a factor affecting the immune response via RBC alloantibodies.
Several studies have reported multi-RBC antigenmatched transfusion and its effects on RBC alloimmunizaiton ${ }^{[18-21]}$. HLA class II typing may improve precision medicine; patients who require transfusion multiple times over a long period may be considered for antigen-matched transfusion depending on the HLA class II typing result. Our study revealed that HLA typing was associated with the presence of RBC alloantibodies; however, our study had a relatively small sample size, and other factors, such as environmental conditions or smoking, must also be taken into account. Large-scale studies or cohort studies are needed to confirm whether HLA-DRB1 alleles have protective effects or are associated with increased risk.

Finally, we concluded that HLA-DRB1 and HLADQB1 alleles were key factors affecting the expression of RBC alloantibodies in Chinese individuals. Our results provided evidence of the associations of HLA-DRB $1 * 01$, HLA-DRB $1 * 17$, HLA-DRB1*09, HLA-DRB $1 * 13$, HLA-DQB $1 * 06$, and HLA$\mathrm{DQB} 1 * 08$ with the presence of RBC alloantibodies.

\section{References}

[1] Verduin EP, Brand A, van de Watering LM, et al. Factors associated with persistence of red blood cell antibodies in woman after pregnancies complicated by fetal alloimmune haemolytic disease treated with intrauterine transfusions. Br J Haematol, 2015,168:443-51.

[2] Schonewille H, Haak HL, van Zijl AM. RBC antibody persistence. Transfusion, 2000,40:1127-31.

[3] Blumberg N, Peck K, Ross K, et al. Immune response to chronic red blood cell transfusion. Vox Sanguinis, 1983,44:212-7.

[4] Fenaux P, Ades L. How we treat lower-risk myelodysplastic syndromes. Blood, 2013,121:4280-6.

[5] Zalpuri S, Zwaginga JJ, LeCessie S, et al. Red-bloodcell alloimmunization and number of red-blood-cell transfusions. Vox Sanguinis, 2012,102:144-9.

[6] Natukunda B, Schonewille H, van de Watering L, et al. Prevalence and specificities of red blood cell alloantibodies in transfused Ugandans with different diseases. Vox Sanguinis, 2010,98:167-71. 
[7] Schonewille H, Doxiadis, II, Levering WH, et al. HLADRB1 associations in individuals with single and multiple clinically relevant red blood cell antibodies. Transfusion, 2014,54:1971-80.

[8] Maluskova A, Mrazek F, Pauliskova M, et al. Association of HLA-DRB1 and HLA-DQB1 with red-bloodcell alloimmunization in the Czech population. Vox Sanguinis, 2017,112:156-62.

[9] Lin YS, Xu ZY, Li XF, et al. Association of HLADRB1 and HLA-DQB1 alleles with red blood cell autoimmunization in the Chinese population. Asia-Pacific Journal of Blood Types and Genes, 2017,1(2):31-4.

[10] Lin YS, Chang JS, Qiu Y, et al. Incidence of unexpected red blood cell antibodies in the north of China. Asia-Pacific Journal of Blood Types and Genes, 2017,1(1):17-24.

[11] Verduin EP, Brand A, Middelburg RA, et al. Female sex of older patients is an independent risk factor for red blood cell alloimmunization after transfusion. Transfusion, 2015,55:1478-85.

[12] Wu Dazhou, Wang MN, Zhou D, et al. Analysis of the relationship between the HLA-DRB1 alleles and the production of RhD antibody. Chin J Blood Transfusion, 2014,12:1290-2.

[13] Verduin EP, Brand A, van de Watering LM, et al. The HLA-DRB $1 * 15$ phenotype is associated with multiple red blood cell and HLA antibody responsiveness. Transfusion, 2016,56:1849-56.

[14] Ovsyannikova IG, Schaid DJ, Larrabee BR, et al. A large population-based association study between HLA and KIR genotypes and measles vaccine antibody responses.
PloS One, 2017,12:e0171261.

[15] Posteraro B, Pastorino R, Di Giannantonio P, et al. The link between genetic variation and variability in vaccine responses: systematic review and meta-analyses. Vaccine, 2014,32:1661-9.

[16] Villabona-Arenas CJ, Mondini A, Bosch I, et al. Dengue virus type 3 adaptive changes during epidemics in Sao Jose de Rio Preto, Brazil, 2006-2007. PloS One, 2013,8:e63496.

[17] Haaland RE, Johnson JA, Tang J. Recent advances in research of HIV infection: implications of viral and host genetics on treatment and prevention. Public Health Genomics, 2013,16 (1-2):31-6.

[18] Makroo RN, Agrawal S, Chowdhry M. Rh and Kell phenotype matched blood versus randomly selected and conventionally cross matched blood on incidence of alloimmunization. Indian J Hematol Blood Transfus, 2017,33(2):264-70.

[19] Delaney M, Wikman A, van de Watering L, et al. Blood group antigen matching influence on gestational outcomes (AMIGO) study. Transfusion, 2017,57(3):525-32.

[20] Gaspardi AC, Sippert EA, De Macedo MD, et al. Clinically relevant RHD-CE genotypes in patients with sickle cell disease and in African Brazilian donors. Blood Trasfus, 2016,14(5):449-54.

[21] Schonewille H, Prinsen-Zander KJ, Reijnart M, et al. Extended matched intrauterine transfusions reduce maternal Duffy, Kidd, and S antibody formation. Transfusion, 2015,55(12):2912-9.

(Received 18 August 2017, Revised 28 August 2017, Accepted 06 September 2017) 